Mechanical Systems and Signal Processing, 18 (4), pp. 849-868, 2004

\title{
Acoustic Emissions diagnosis of rotor-stator rubs using the KS statistic
}

\author{
L. D. Hall, D. Mba \\ School of Engineering, Cranfield University, Bedfordshire, UK, MK43 OAL
}

\begin{abstract}
Acoustic emission (AE) measurement at the bearings of rotating machinery has become a useful tool for diagnosing incipient fault conditions. In particular, AE can be used to detect unwanted intermittent or partial rubbing between a rotating central shaft and surrounding stationary components. This is a particular problem encountered in turbines used for power generation. For successful fault diagnosis, it is important to adopt $\mathrm{AE}$ signal analysis techniques capable of distinguishing between various types of rub mechanisms. It is also useful to develop techniques for inferring information such as the severity of rubbing or the type of seal material making contact on the shaft.
\end{abstract}

It is proposed that modelling the cumulative distribution function of rub-induced AE signals with respect to appropriate theoretical distributions, and quantifying the goodness of fit with the KolmogorovSmirnov (KS) statistic, offers a suitable signal feature for diagnosis. This paper demonstrates the successful use of the KS feature for discriminating different classes of shaft-seal rubbing. 
Mechanical Systems and Signal Processing, 18 (4), pp. 849-868, 2004

\section{Introduction}

Measurement of high frequency acoustic emissions (AE) has become a viable technique in the condition monitoring of many types of rotating machinery [1-5]. On in real operational machinery it is often only practical to take AE measurements from non-rotating members, at or on the bearing housing. Consequently, AE signals originating from the rotating shaft will incur significant attenuation across the transmission path to an $\mathrm{AE}$ receiver attached at the bearing housing. This can be related to inhomogenities and scatterers within the structure, reflections at acoustic boundaries, interference and attenuation effects across the bearing interfaces. Moreover, the AE signal will be further coloured by the characteristic frequency response of the AE transducer itself. In light of these factors, interpretation of the AE signals is not trivial and often departs from the classic AE signal model $[6,7]$.

In recent years various signal processing and pattern recognition techniques have been successfully applied to AE signals for diagnosing the severity and location of defects in various types of rotating machinery. Notably artificial neural networks (ANN) [8] and clustering [4,9] have been adopted for AE signal classification. Regardless of the classification engine employed, it is invariably identification of the key resolving features or descriptors within the $\mathrm{AE}$ signal that is paramount for successful classification. Generally, the size of the feature vector chosen depends upon the specific application and recognition requirements. Previous studies [10] employed relatively large feature vectors for the AE signal classification problem. However, for discriminating between different classes of rub signatures, it was considered useful to define a feature vector with a minimum number of parameters.

Typical features extracted from AE signatures in condition monitoring include peak or total energy, standard deviation, median, AE counts, r.m.s voltage and duration. However, these are all related to 
Mechanical Systems and Signal Processing, 18 (4), pp. 849-868, 2004

absolute energy levels of the measured waveform or rely upon pre-set amplitude thresholds. As such, the quantities exhibit considerable variability from one bearing measurement to the next and are thus extremely dependent upon factors such as background noise, in addition to AE transducer positioning and coupling. Consequently, it is believed that such features are not ideal for AE waveform classification, especially in cases where several measurement positions are required. Alternative features more related to the amplitude statistics of the measured AE waveforms and independent of absolute energy levels have also been considered. Notably, the fourth statistical moment known as kurtosis and the ratio of peak to r.m.s voltage known as crest factor have been applied for condition monitoring in rotating machinery. However, laboratory tests conducted as part of this research have indicated both of these quantities to be unsuitable for classification.

In contrast, it has been shown that modelling an AE signal as an autoregressive stochastic process, as described by Melton [11] and later Mba [4], can provide good AE classification results. However, the use of AR coefficients as signal features approximating the shape of the signal has some disadvantages. Primarily, it is always necessary to determine the number of AR coefficients necessary to adequately represent each AE signal. Although numerous algorithms exist for determining the model order, it should be noted that the classification results could be sensitive to the AR model order. Secondly, it was evident from this study that AE signal classification using AR model coefficients was severely impaired when the measured AE signals was modulated by small levels of background acoustic noise.

In light of this discussion, it is postulated that a robust AE signal feature based upon amplitude statistics and independent of absolute energy levels or pre-defined thresholds, and less effected by pre-signal processing, can be a useful addition to AE signal classification. This paper proposes that the standard Kolmogorov-Smirnov statistic can provide such an AE waveform feature parameter for classifying 
Mechanical Systems and Signal Processing, 18 (4), pp. 849-868, 2004

different types of rubbing in rotating machinery. To demonstrate this, classification results are presented from rub experiments conducted on a journal bearing test rig that rotates at $1500 \mathrm{rev} / \mathrm{min}$ (rpm). In addition, classification performance of this technique was tested by modulating the measured AE signals with background noise taken from bearings of an operational 550 MW turbine unit.

\section{Acoustic Emission and rubbing in rotating machinery}

Fundamentally, a light frictional rub between the central shaft and surrounding stationary components, such as the seals within a turbine, will cause microscopic perturbation and a transient release of broadband strain energy referred to as Acoustic Emissions. Although originating from a different mechanical process, this wave motion is in practice very similar to the wave energy that propagates from microscopic cracks within solid structures. A number of reasons can be identified for the onset on light rubbing in a rotating plant. These include thermal effects, foundation movement, component movement, rotor unbalance or misalignment. Regardless of the exact relationship between cause and effect, the existence of rubbing is unwanted as it can often develop into more significant mechanical distress. Two main categories of light rubbing can be identified. Primarily partial rubbing constitutes distinct or intermittent rub events occurring instantaneously within the period of the shaft rotation. Secondly continuous rubbing involves more sustained contact between shaft and surrounding components. Although the mechanisms by which rub phenomena escalate are complex, it is suggested that for machinery of higher rotational speeds, a high concentration of partial rub events can lead to more sustained rubbing, which in turn can induce more serious vibration via mechanisms such as thermal bending of the shaft. Consequently, it is considered in this paper that an AE system capable of diagnosing individual partial rub events might promote the early diagnosis of impending mechanical distress. 
Mechanical Systems and Signal Processing, 18 (4), pp. 849-868, 2004

Sato [2] reported that $\mathrm{AE}$ measurement can provide a valuable complementary tool for diagnosing rubbing in fast rotating plant such as turbine generators. For this investigation, signatures were measured at frequency bands greater than $100 \mathrm{kHz}$, overcoming much of the mechanical background noise whilst increasing the probability of direct rub detection. To detect rubbing using AE, it is generally advantageous to minimise both the physical distance and number of interfaces between the location of rubbing and the AE receiver. This is because significant acoustic attenuation will occur, especially at higher frequencies (>100 kHz), due to frequency-dependent absorption, geometric spreading losses and reflection at interfaces. In some slow rotating cases, it is possible to place transducers directly on the rotating shaft, [4]. However in many types of operational rotating machinery, such as in turbinegenerators, it is only practical to make measurements remotely at the bearing housing. In such cases, $\mathrm{AE}$ signals produced by rubbing on the shaft will incur considerable attenuation as the signatures propagate along the shaft surface to the bearing, across an oil film and into the bearing housing. This attenuation issue is considered to be the limiting factor for $\mathrm{AE}$ in many examples of large-scale machinery. 
Mechanical Systems and Signal Processing, 18 (4), pp. 849-868, 2004

\section{Theory}

Figure 1 shows an example AE signal measured at the bearing housing of a test rig whilst rotating at $1500 \mathrm{rpm}$. This signature was a result of simulating a partial rub on the shaft with a steel seal fixture. Clearly, the AE burst shows some resemblance to the shape of a classic AE waveform produced by crack propagation or a Hsu-Nielson source, although it is not possible to identify individual extensional and flexural wave modes as can be often obtained by the Modal AE approach in thin plates. A notable feature of this rub waveform is the initial onset of acoustic energy through the succession of initial high energy peaks prior to the exponential decay. Therefore, it might be inferred that useful signal features might include kurtosis or crest factor as they describe the extreme values in the amplitude distribution. However, investigation of numerous rub signatures has shown considerable inconsistency in these parameters under stable experimental conditions. Consequently, they are not considered suitable parameters for classifying rubbing.

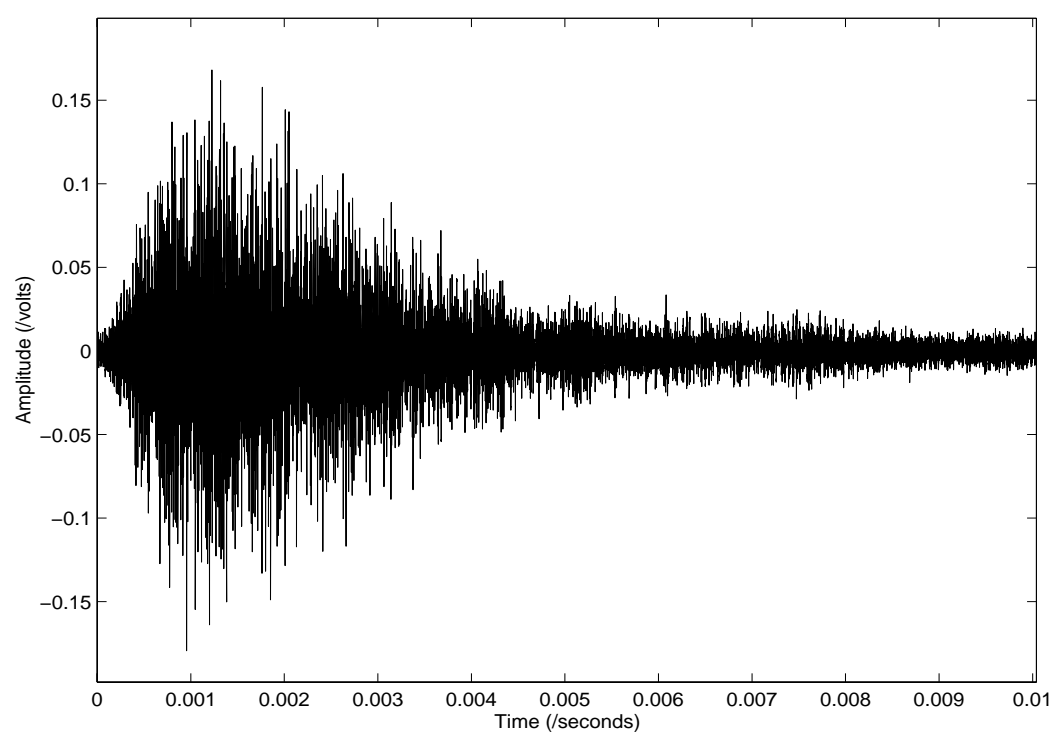

Fig 1 A shaft-seal rub induced AE signal measured at the journal bearing of a test rig. 
Mechanical Systems and Signal Processing, 18 (4), pp. 849-868, 2004

Alternatively, it is conceived that the Kolmogorov-Smirnov (KS) test statistic [12] might provide a more appropriate feature vector for classifying AE rub signals. In essence, this standard goodness-of-fit test quantifies the difference between the amplitude statistics of the measured AE signal and a specified theoretical distribution function model. It is defined as the maximum absolute difference (D) between the empirical cumulative distribution function $\mathrm{S}_{\mathrm{N} 2}(\mathrm{x})$ and a hypothesised theoretical distribution function $\mathrm{S}_{\mathrm{N} 1}(\mathrm{x})$, see equation (1)

$$
D=\max _{-\infty<x<\infty}\left|S_{N 1}(x)-S_{N 2}(x)\right|
$$

As $\mathrm{S}_{\mathrm{N} 1}(\mathrm{x})$ and $\mathrm{S}_{\mathrm{N} 2}(\mathrm{x})$ are non-decreasing and $\mathrm{S}_{\mathrm{N} 2}(\mathrm{x})$ is considered to be constant between the defined values of amplitude $\mathrm{x}$, as illustrated in Fig. 2, the maximum deviation between the two curves will occur at one of the defined observation points $\mathrm{x}_{1}, \mathrm{x}_{2}, \mathrm{x}_{3} \ldots \mathrm{x}_{\mathrm{n}}$. It should also be noted that the distribution of $\mathrm{D}$, in the null hypothesis, can be calculated to give the significance of any observed non-zero value of D. Moreover the statistic is invariant under re-parametrisation of x. For example, the same significance exists under $\mathrm{x}$ as for $\log (\mathrm{x})$. 


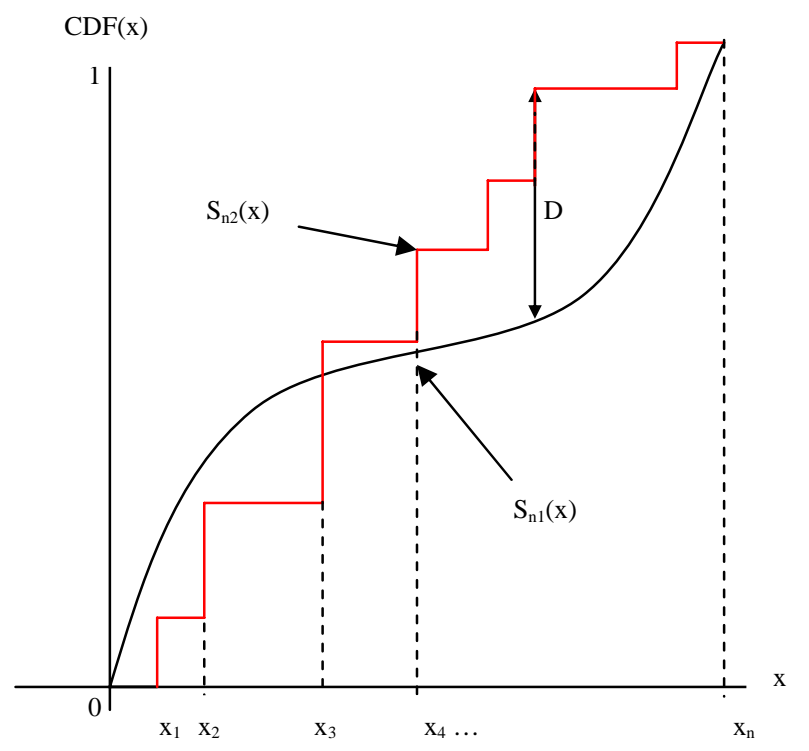

Fig. 2 'D' is the greatest distance between an empirical distribution $\mathrm{S}_{\mathrm{n} 2}(\mathrm{x})$ and the theoretical distribution model $\mathrm{S}_{\mathrm{n} 1}(\mathrm{x})$.

The basic algorithm devised for this paper is summarized in figure 3. Primarily, each digital AE signal was decimated by a factor of 5 to reduce the KS computation time. This decimation was not expected to degrade classification results because of the high initial sampling rate used in the analogue-to-digital convertor (ADC) (4 MHz). Following this stage, theoretical distribution models for each of the AE signals were derived, assuming the null hypothesis that each signal belongs to the candidate family of distributions. These theoretical cumulative distribution function (CDF) curves were derived directly by the method of moments as opposed to a maximum likelihood approach. For example, for the Gaussian model, the mean $\mu$ and variance $\sigma^{2}$ of each input signal was determined and the model estimate of the CDF evaluated using the two parameter given in equation (2). Other families considered included the Rayleigh and exponential distribution families, as depicted in figure 3. From this, KS values for each 
Mechanical Systems and Signal Processing, 18 (4), pp. 849-868, 2004

signal with respect to the selected distribution families were evaluated and used as the input feature vectors for the classifier.

$$
f(x \mid \mu, \sigma)=\frac{1}{\sqrt{2 \pi} \sigma} \int_{-\infty}^{x} e^{\frac{-(t-\mu)^{2}}{2 \sigma^{2}}} d t
$$

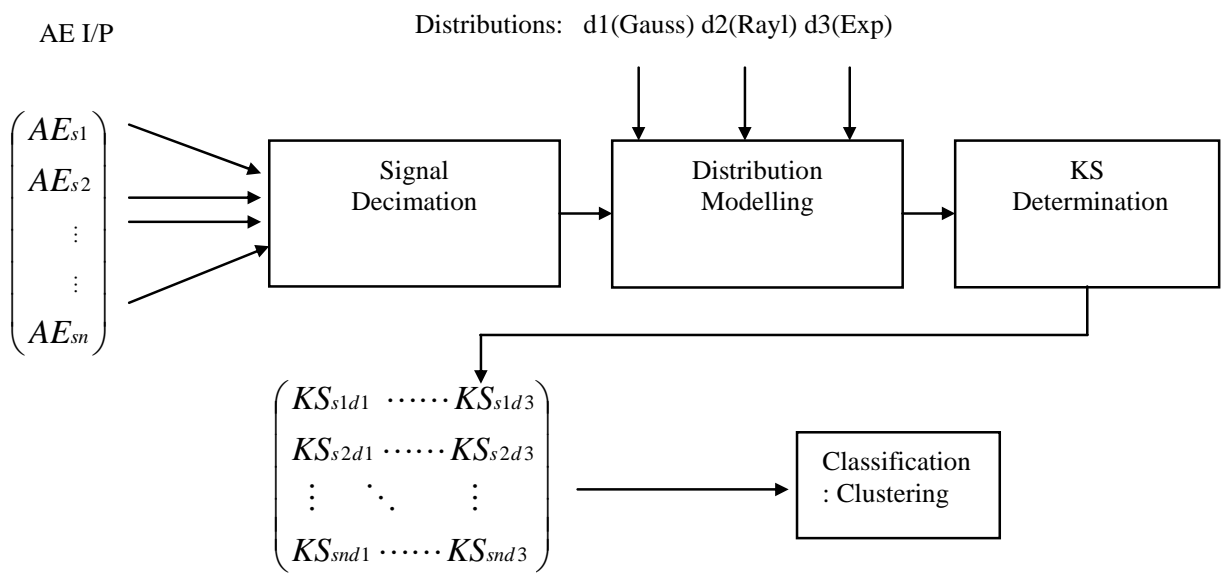

Fig. 3 A schematic for the AE classification algorithm

The classifier adopted for rub signal classification was a hierarchical clustering algorithm [10, 13-15], although it is believed that other approaches, notably k-means or even neural network approaches, could equally demonstrate the use of the KS descriptor. Clustering describes the action of partitioning a set of objects into natural groups so that the profile of the objects in the same cluster are very similar and the profiles of objects in different clusters are quite distinct. It is proposed that AE signatures from the same rub category (e.g. partial steel rubbing, partial brass rubbing, etc.) could be clustered in well-defined groups. This involves sequential stages of:

1. Similarity, in which the Euclidean distance between every pair of KS signal vector was determined within the similarity matrix and 
Mechanical Systems and Signal Processing, 18 (4), pp. 849-868, 2004

2. Binary Linkage; in which a series of binary clusters of increasing size are made using the information in the similarity matrix, starting with the closest two signal objects, until all the objects are linked together in a hierarchical tree.

Clustering results are represented graphically in this paper using an agglomerative dendrogram plot, in which individual signals are labelled on the x-axis whilst the distance between the centroids of clusters are shown on the y-axis. The classification procedure described was used to assess whether KS features associated with $\mathrm{AE}$ signatures was sufficiently robust to cluster known rub classes into the correct natural groups.

\section{Experimental set-up}

A Physical Acoustic Corporation wideband (WD) piezoelectric sensor was employed for this work with a range of $100 \mathrm{kHz}-1000 \mathrm{kHz}$. Pre-amplification ranged from 40 to $60 \mathrm{~dB}$. The signal output from the pre-amplifier was connected (i.e. via BNC/coaxial cable) directly to a commercial data acquisition card that occupies one of the ISA slots within a Pentium host PC. This AEDSP acquisition card provided up to $8 \mathrm{MHz}$ sampling rate and incorporated 16-bit precision giving a dynamic range of more than $85 \mathrm{~dB}$. Prior to the analog-to-digital converter (ADC), the card employed anti-aliasing filters that can be controlled directly in software. Moreover, an extended local memory allowed the sequential recording of signals containing up to 256,000 samples. This corresponded to the continuous measurement over more than $0.06 \mathrm{~s}$ at a sampling rate of $4 \mathrm{MHz}$.

Figure 4 (a) depicts the rotating test rig. As shown, the shaft is supported by two journal bearings. An acoustic wave-guide made contact with the inner bearing housing and the receiving $\mathrm{AE}$ sensor was attached to the other end, ensuring a direct transmission path. Throughout the experimentation high temperature acoustic couplant was applied to ensure a good acoustic contact between transducer and the 
Mechanical Systems and Signal Processing, 18 (4), pp. 849-868, 2004

wave-guide and between wave-guide and the bearing housing. The rub simulation mechanism was positioned between the third and fourth of five steel discs shrink-fitted on to the central shaft. Figure 4 (b) illustrates how partial rubs are simulated using this device. Essentially, a non-concentric dummy shaft fixture is attached to the rotor so as to rub against a supported seal fixture on every shaft rotation. The seal fixtures employed were machined in mild steel or brass. The reaction pressure exerted by the seal upon the incident shaft was set by masses applied to the rub fixture as shown in Fig. 4(b). These masses applied a force of approximately $140 \mathrm{~N}$ throughout the measurements.

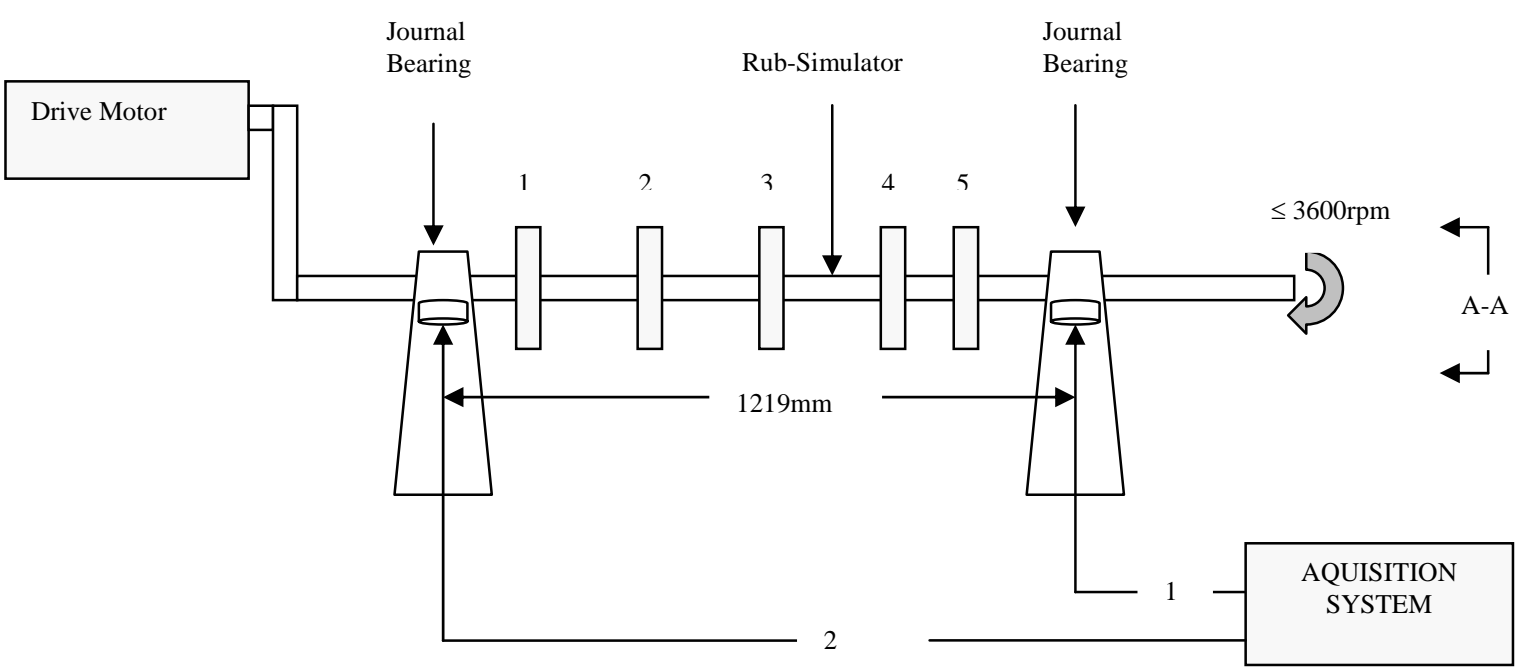

(a)
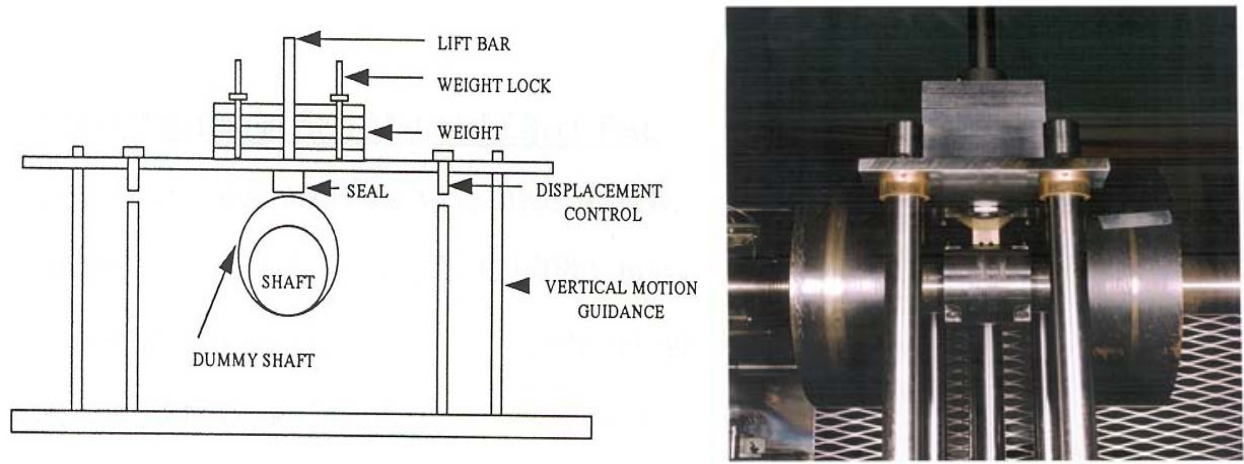

(b)

Figure 4 (a) The journal bearing test rig, (b) The rub simulation mechanism. 
Mechanical Systems and Signal Processing, 18 (4), pp. 849-868, 2004

\section{Results and discussion}

Using the aforementioned system, AE signals were recorded for different types of partial shaft-seal rubbing. Primarily signals from the partial rubbing of steel and brass seal fixtures were taken. Secondly, partial rub signals were taken from three steel seal fixtures exhibiting different states of wear. Finally, rub signatures were taken from three seal fixtures with faces of different geometric shape. This section presents results pertaining to the use of the KS statistic to classify the defined rub classes by employing hierarchical clustering. By comparing the results to alternative AE feature extraction methods, the potential of the KS statistic is demonstrated.

It must be noted that identically shaped brass and steel seal fixtures were employed, see figure 5. In each case, fifteen AE signatures are recorded under the same experimental conditions, i.e., the shaft period and the load applied to the rub simulation mechanism remained constant. Examples of the AE signals produced from steel and brass seal rubbing are shown in figure 6.
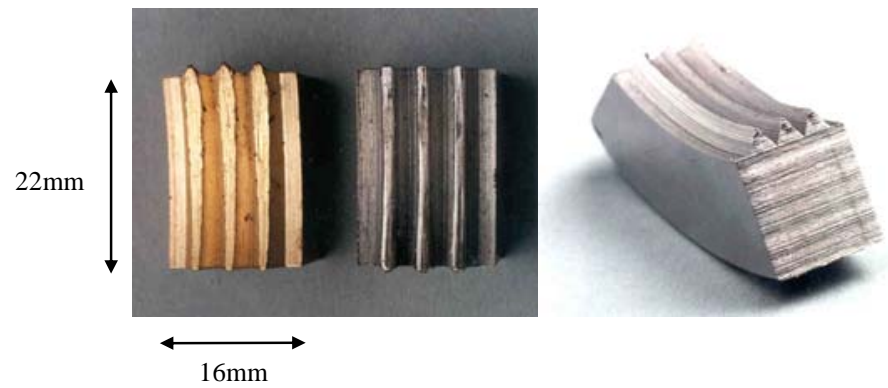

Figure $5 \quad$ Steel and brass seal fixtures 
Mechanical Systems and Signal Processing, 18 (4), pp. 849-868, 2004

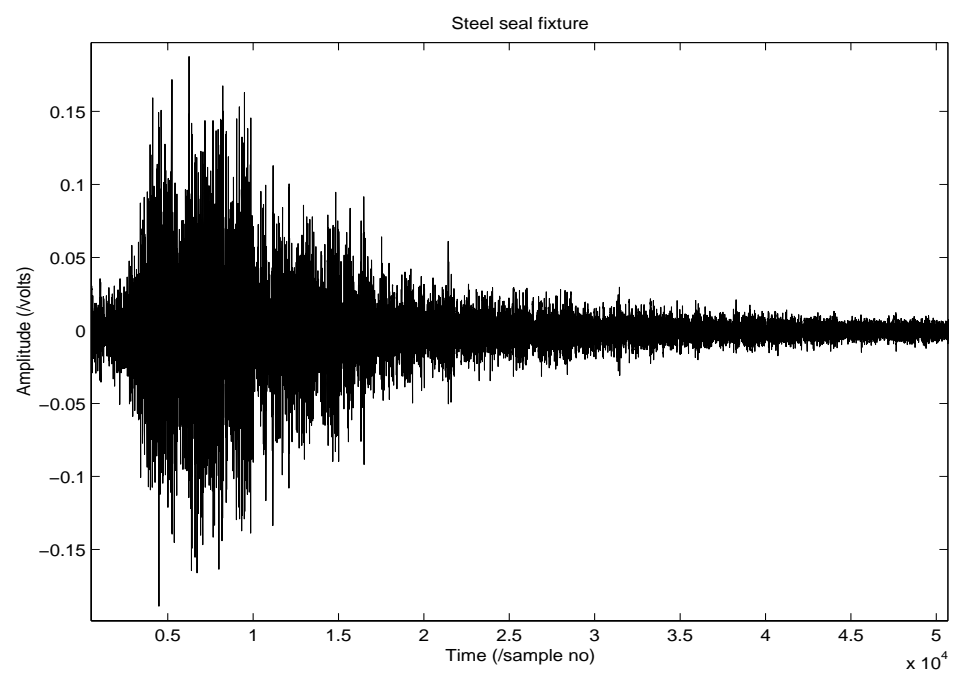

(a)

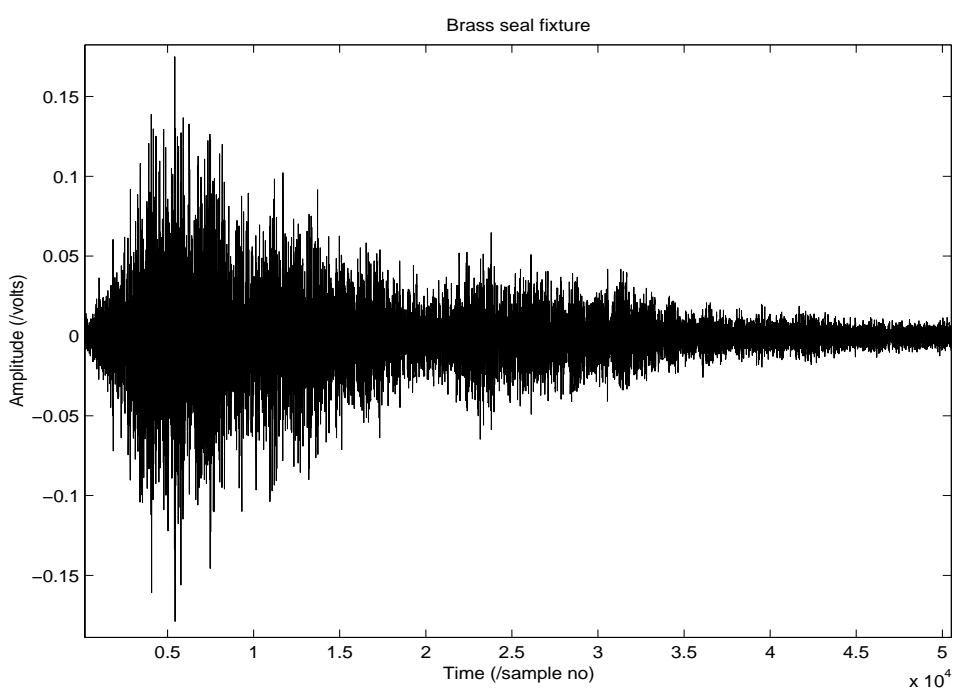

(b)

Figure $6 \quad$ Examples of the AE signals produced using (a) steel and (b) brass seal fixture

Appendix A lists mean and standard deviation values for extracted AE features for the example steel and brass signals. The KS values were obtained using the Gaussian, Rayleigh and exponential distribution families. Gaussian KS values for each of the signals multiplied by two were also shown and it should be noted that these values are not significantly different to the Gaussian KS values obtained from the 
Mechanical Systems and Signal Processing, 18 (4), pp. 849-868, 2004

original signals. Although many established AE signal features were considered, only the r.m.s, median, kurtosis and autoregressive (AR) coefficients are detailed as they proved more significant rub indicators.

Primarily, use of the KS values derived from the Gaussian model for all thirty signals are considered as the input vector to the clustering algorithm. The achieved clustering results are depicted using the dendrogram plot in figure 7. As shown, the x-axis represents the steel fixture rub signals by labels 1 to 15 and the signals produced using the brass fixture as signals 16 to 30. Moreover, the $y$-axis shows the Euclidean distances between the input KS values. It is evident that the two main clusters discriminate between the brass and steel rub classes. Moreover, ignoring signal-6 and signal-12 corresponding to steel rubbing, the separation is relatively pronounced.

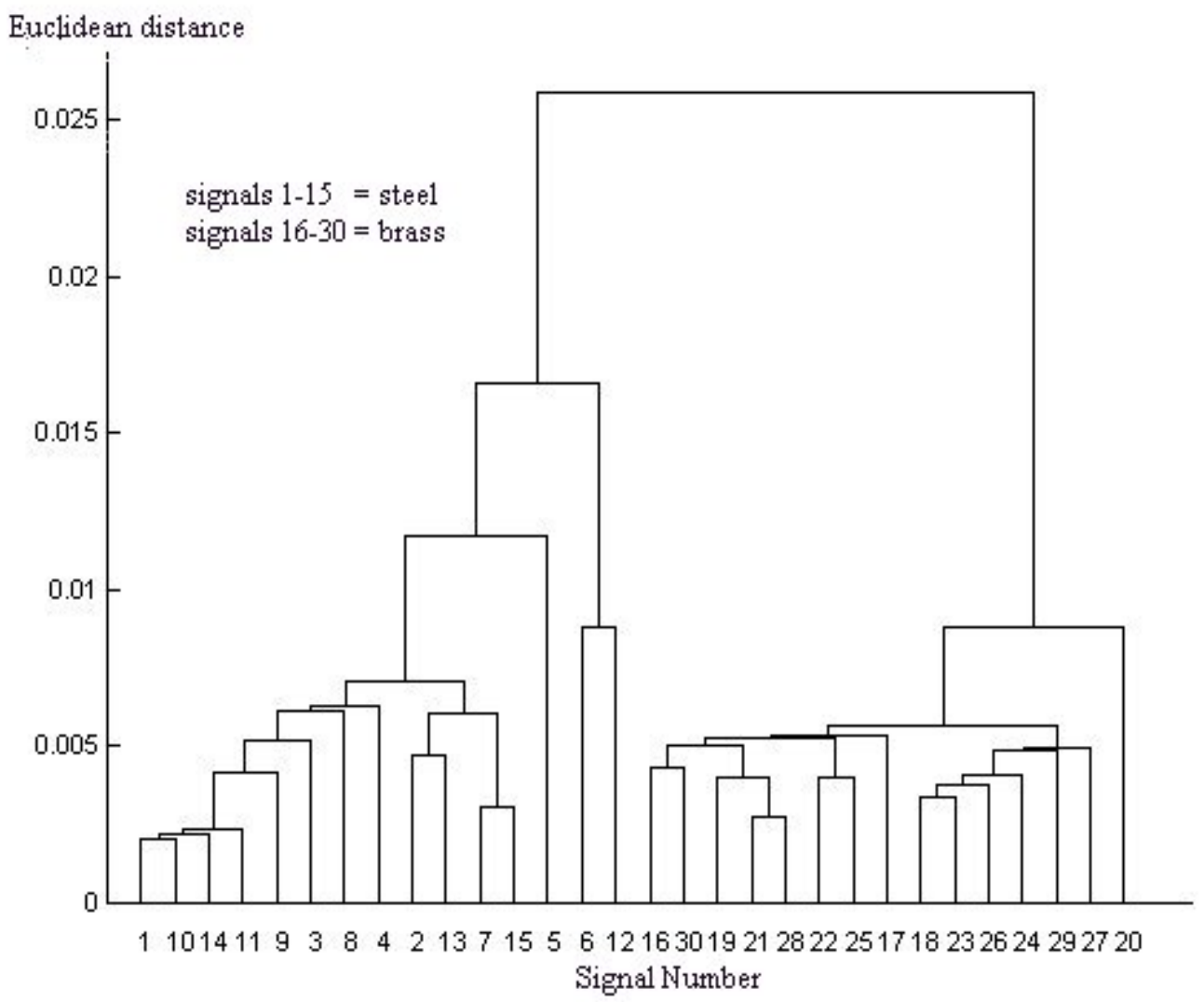

Figure 7 Gaussian KS discrimination, single-linkage (nearest neighbour) 
Mechanical Systems and Signal Processing, 18 (4), pp. 849-868, 2004

The algorithm used to determine KS values involves the generation of a random reference signal and hence produces slight variation in the $\mathrm{KS}$ value for a given $\mathrm{AE}$ signal over repeated calculation. However, this variability did not prevent adequate separation between brass and steel signals over many independent experiments. Moreover, it was apparent that using a signal feature vector that constitutes repeated KS evaluations for a specified reference distribution could improve the clustering results. Figure 8 shows the clustering achieved using a feature vector consisting of four independent KS evaluations assuming the Gaussian model. In this plot, Ward's method of clustering is employed in preference to the single-linkage (i.e., nearest neighbor). This is a procedure in which the similarity used to join two clusters is calculated as the sum of squares between the two clusters summed over all variables. It is effective in increasing the 'within cluster' homogeneity and prevents chaining.

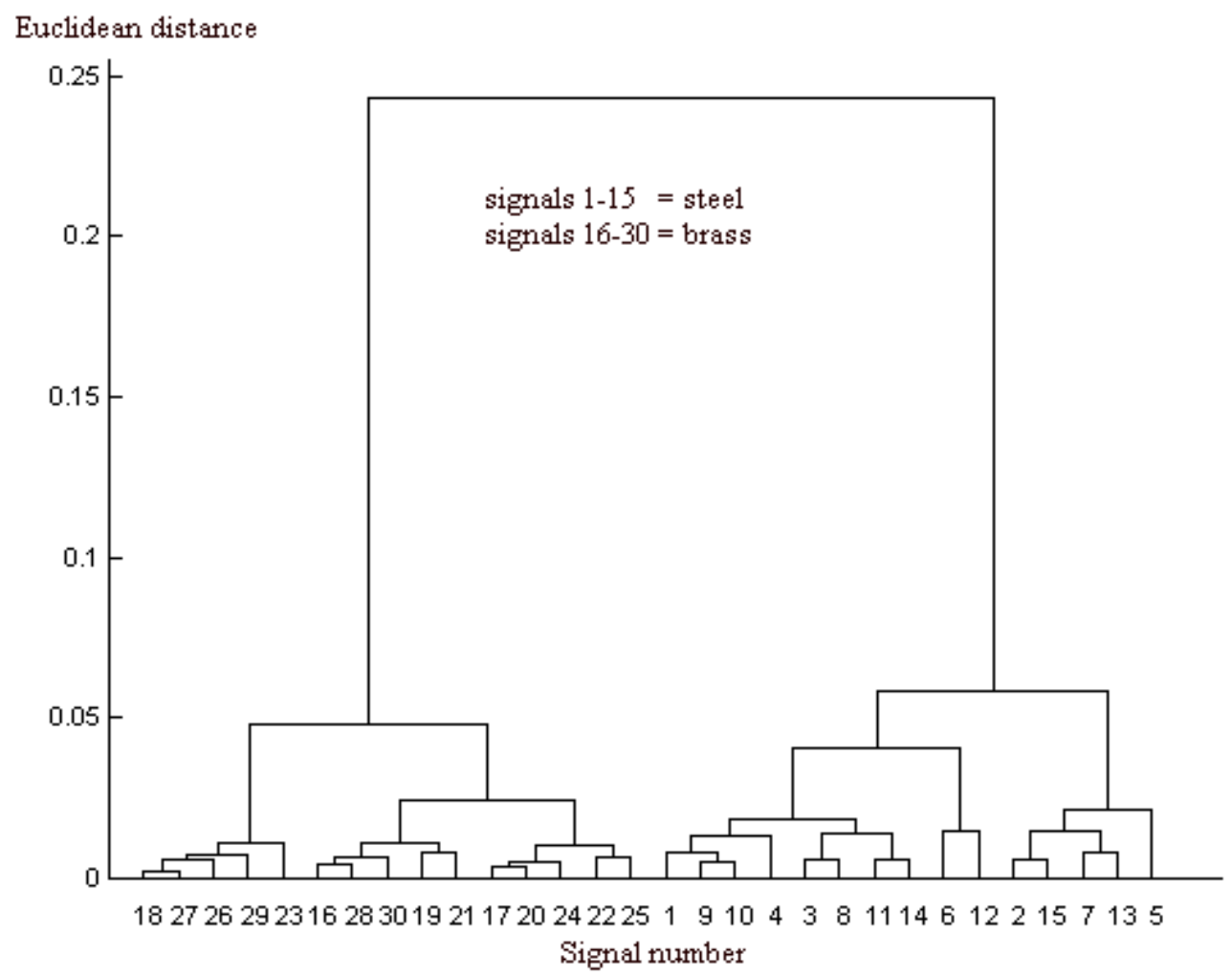

Figure 8 Gaussian KS discrimination, Ward's method 
Mechanical Systems and Signal Processing, 18 (4), pp. 849-868, 2004

In contrast to the robust KS results, clustering of the thirty partial rub signals using established $\mathrm{AE}$ signal features such as peak energy, total energy, R.M.S, median, standard deviation, counts and peaks within an FFT revealed poor separation between brass and steel signals. The most appropriate of such features was the signal kurtosis. This energy independent measure of signal spikiness generally yielded larger values for steel rubbing signals than from brass rubbing. However, repeated tests revealed a number of spurious clustering results. This was attributed to the large spread of kurtosis values obtained across all of the AE signals measured.

Although the KS technique outperformed established signal features dependent upon energy or extreme values within the amplitude distribution, it is noted that application of autoregressive coefficients as input AE signal features also produced effective discrimination between brass and steel rubbing. This technique [16] involves modelling each AE signal as an $\mathrm{N}^{\text {th }}$ order stochastic process and using the subsequent AR coefficients within the clustering algorithm to represent the signal 'fine-scale' shape to within a certain accepted prediction error. The optimum order $(\mathrm{N})$ for the AR model is often the lowest order at which the minimum mean-squared error becomes stationary and can be determined via the application of the final prediction error (FPE) and Akaike's information criterion (AIC) $[17,18]$. However, it was considered that the success of subsequent AE signal clustering was extremely sensitive to the number of AR coefficients employed, as the optimum order varied for different classes of rub signal. In addition to this draw back, it became clear that the AR approach to partial rub classification breaks down when the measured rub signals are modulated by background acoustic noise.

To demonstrate the effect that background noise can have upon rub classification, acoustic noise from the bearings of an operational $500 \mathrm{MW}$ turbine unit was added to the brass and steel rub signals. Figure9 shows hierarchical clustering results achieved from fifteen noisy brass signals and fifteen noisy steel 
Mechanical Systems and Signal Processing, 18 (4), pp. 849-868, 2004

signals using a $20^{\text {th }}$ order Autoregressive model as the signal descriptors. This clearly demonstrates that the AR approach becomes inappropriate for brass and steel classification when even low levels of background noise are present. In contrast, it became clear that KS-based rub classification is less affected by the addition of real noise. Figure-10 depicts a typical clustering result using the KS algorithm described earlier. In this case, the same levels of turbine noise was added to the thirty rub signals and the same bandpass filter was applied. As shown, AE signal classification using KS descriptors is more suitable than that based upon the more established AR technique.

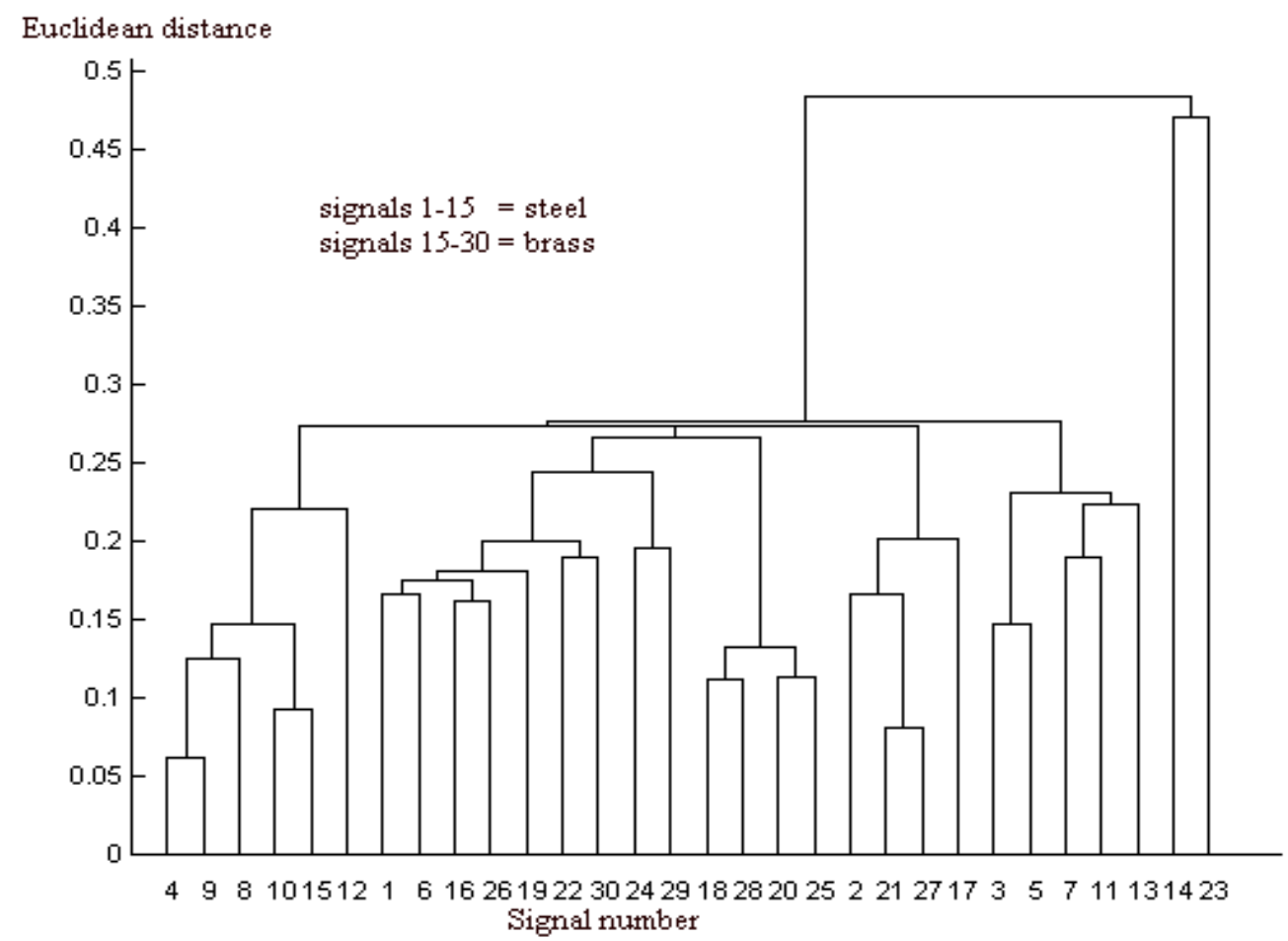

Figure $9 \quad \mathrm{AR}(20)$ discrimination with low levels of real noise measured from a 500MW turbine 
Mechanical Systems and Signal Processing, 18 (4), pp. 849-868, 2004

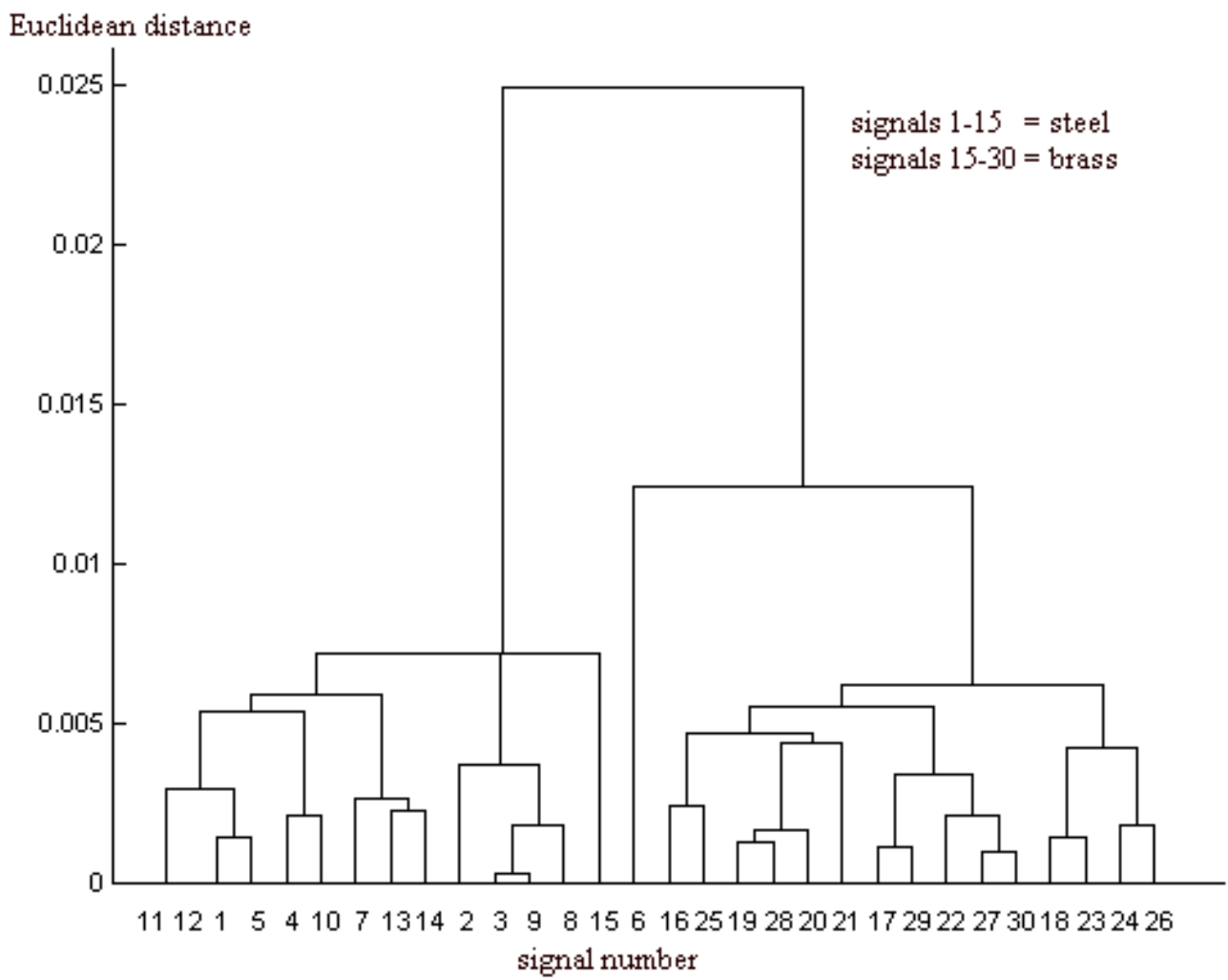

Figure 10 KS discrimination with low levels of real noise measured from a 500MW turbine.

Secondly, it was proposed that some diagnosis of the 'wear state' of rubbing seals might be inferred from AE measurement at the bearings. Three mild steel V-groove seals at different stages of wear were prepared. These are shown in Fig. 11 and are referred to as unworn, mid-wear and worn. Applying each of these fixtures to the partial rub simulator in turn, AE signatures were recorded at the bearings. Figure 12 shows examples of the signatures obtained for the three wear states at the bearing- 1 . By inspection, it is obvious that the unworn signal appears to be more complex than the other two wear state signals. Specifically, it is clear that two smaller bursts appear in the tail of the unworn AE waveform. However, visually identifying the difference between the mid-wear and worn state is more difficult, especially as the peak amplitudes of all three are very similar. 
Mechanical Systems and Signal Processing, 18 (4), pp. 849-868, 2004

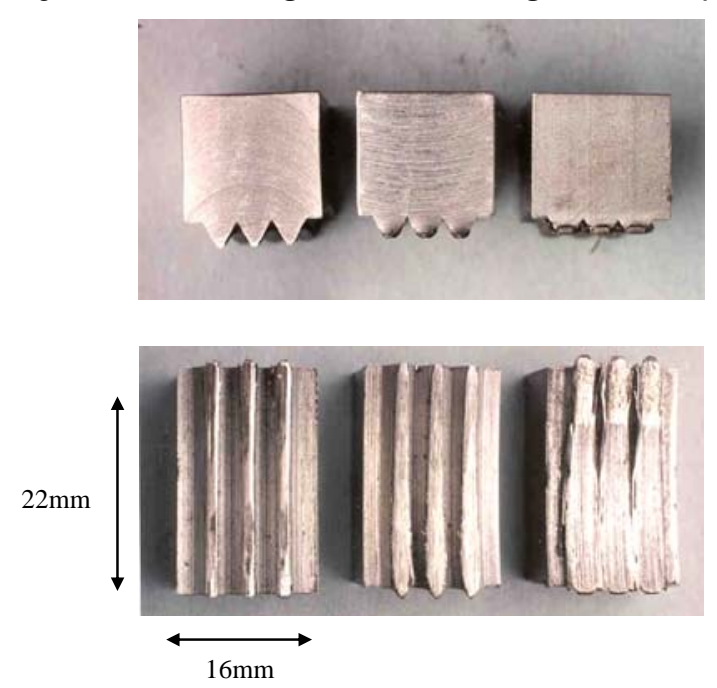

Figure 11 Unworn, mid-wear and worn steel seal fixtures

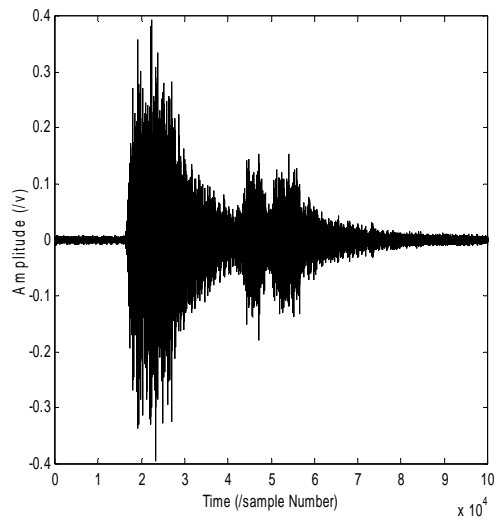

(a)

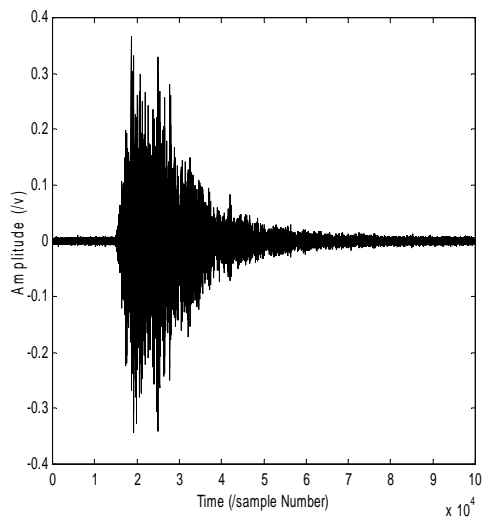

(b)

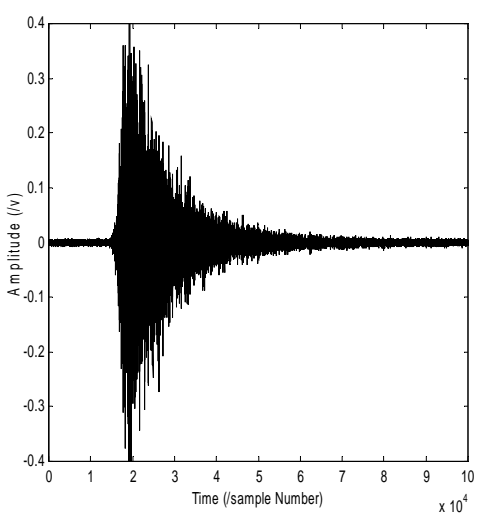

(c)

Figure 12 Example AE signals from a V-Grooved steel seals exhibiting

(a) No wear (b) Mid-wear (c) Extreme wear

To illustrate an automatic classification approach for these wear states, fifteen example signals were taken; five from each of the wear states. Initially, the AR model approach to clustering was applied. Inspection of the residual error plot, using the Akaike's Information Criterion, suggested that an AR order of 8 was sufficient to represent the signature shape. To test the applicability of the AR approach, a plot of the clustering achieved by measuring Euclidean distances between centriod values of AR coefficients associated with each signature is shown in figure14. No pattern in the cluster was observed 
Mechanical Systems and Signal Processing, 18 (4), pp. 849-868, 2004

and it was deduced that the AR classification approach is not suitable for determination of the wear state of the rubbing seal.

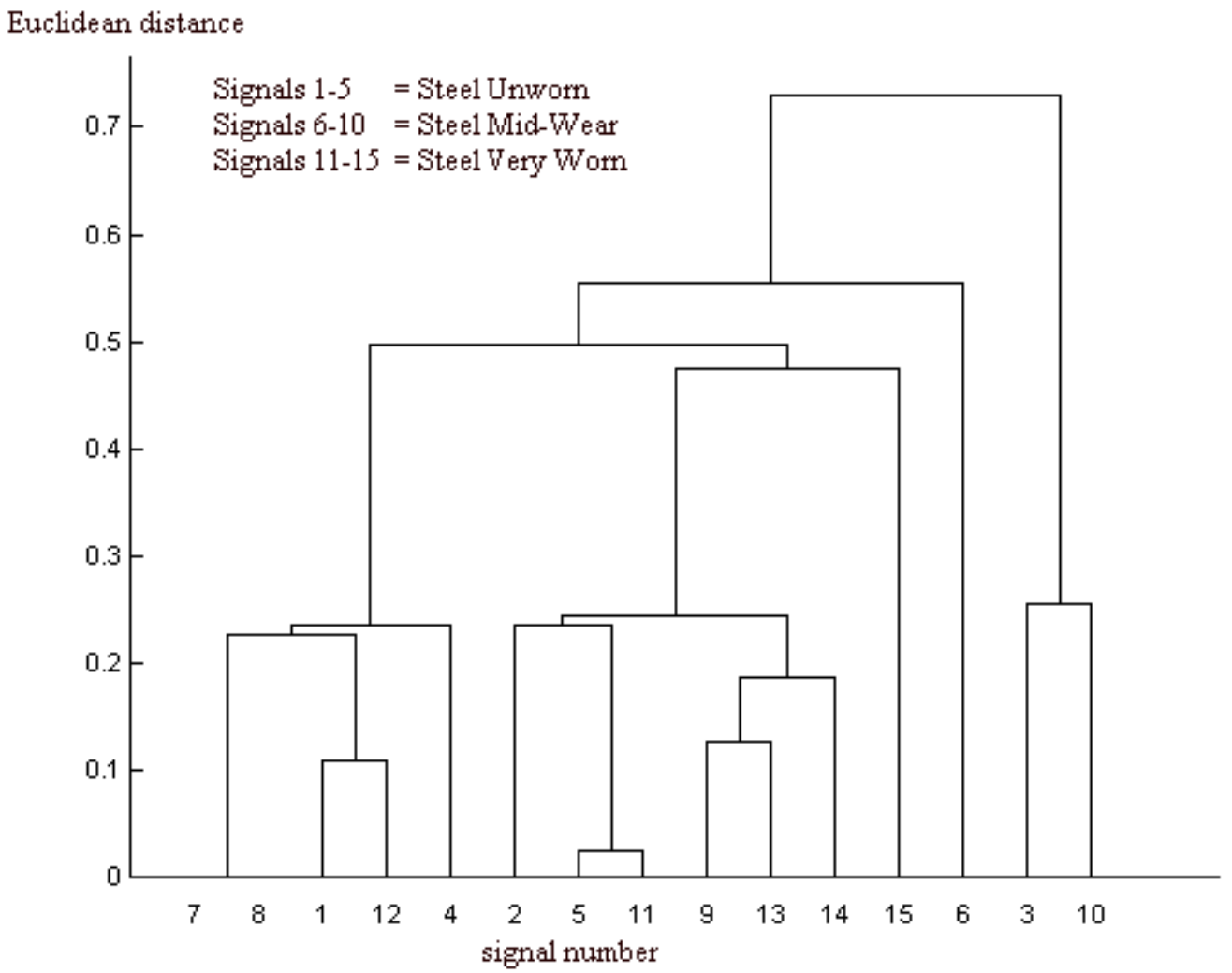

Figure 14 Clustering achieved using 8 AR coefficients for unworn (signals 1-5), mid-wear (signals 6-10) and very worn (signals 11-15) shaft-seal rubbings.

To investigate using the KS statistic for distinguishing between the unworn, mid-wear and worn partial rubbing states, the same fifteen signals were used. Only the Gaussian distribution was used as the reference within the KS algorithm and the cluster result can be seen in figure 15. It is clear from this that reasonably good wear classification has been achieved. Primarily, the two major clusters discriminate between the unworn AE signals and the other two types. This may be justified in that the second and third smaller bursts within the unworn signals cause significant deviation from Gaussian amplitude 
Mechanical Systems and Signal Processing, 18 (4), pp. 849-868, 2004

statistics. Secondly, the Gaussian KS statistic proved sensitive enough to separate mid-wear and very worn rubbing signals within the broader cluster group.

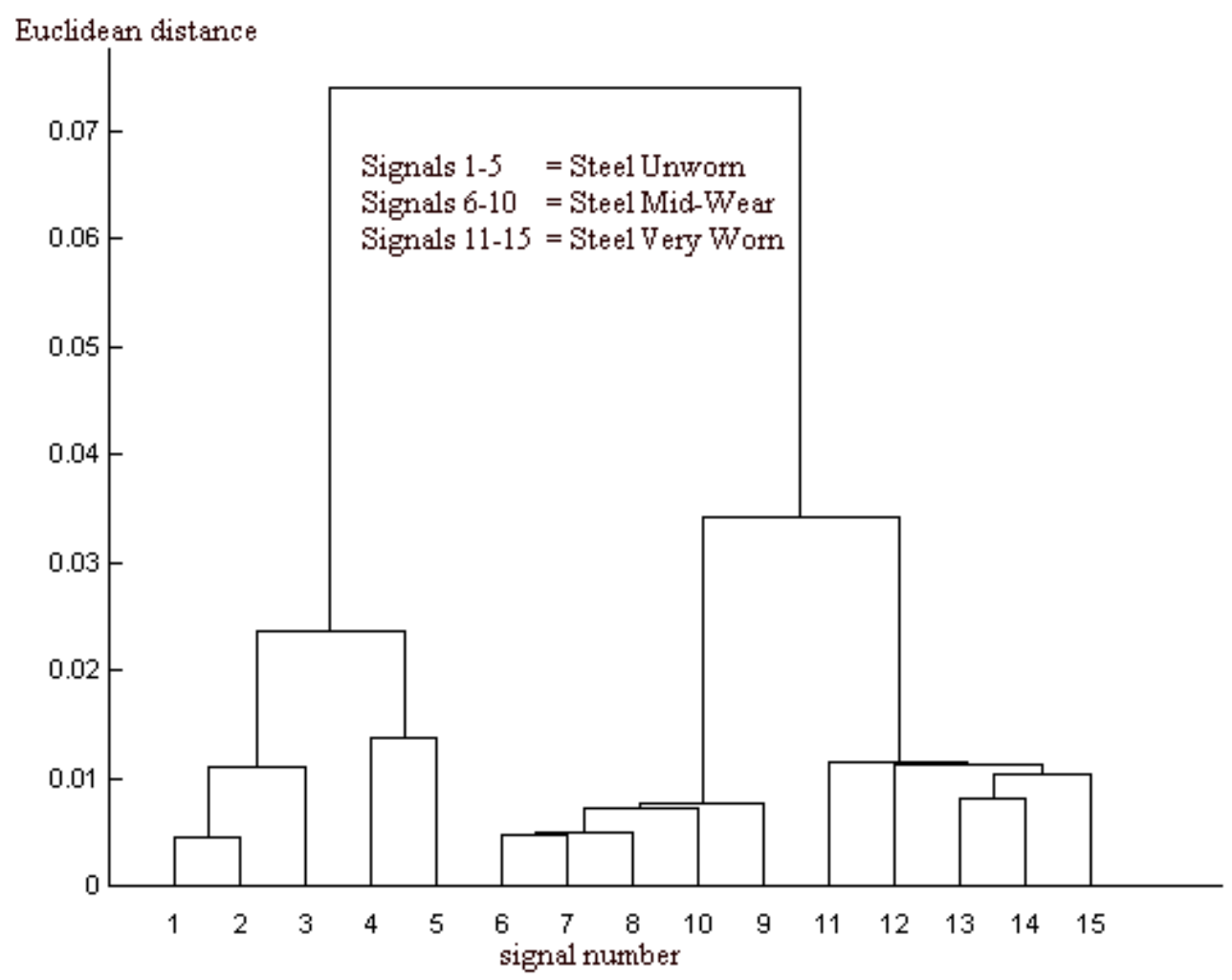

Fig. 15 Clustering achieved using the KS-statistic for unworn (signals 1-5), mid-wear(signals 6-10) and very worn(signals 11-15) shaft-seal rubbings.

Finally, it was of interest to investigate whether the AE technique could distinguish between partial seal rubs from different shapes of seal, machined from the same material. This is because the seal and gland tip geometry within operational turbines will often differ depending upon the desired radial clearance. The seal shapes considered for this study (figure 16) were all machined from the same grade of mild steel and are known as two-grooved flat labyrinth (2GFL), one-grooved flat labyrinth (1GFL) and the two-grooved knife-edged (2GKL) seals. Examples of the three classes of signature measured at bearing2 are shown in figure 17. 
Mechanical Systems and Signal Processing, 18 (4), pp. 849-868, 2004

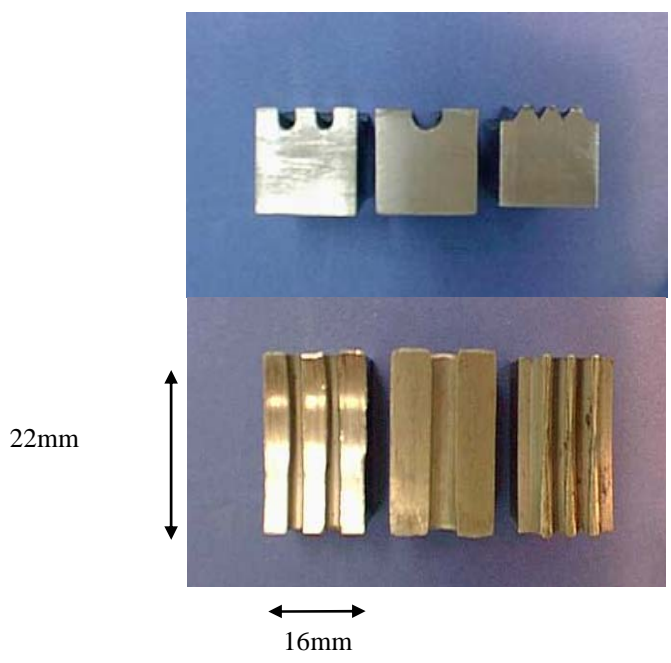

Figure 16: (a) Steel 2GFL seal (b) Steel 1GFL seal (c) Steel 2GKL seal fixtures after experimentation

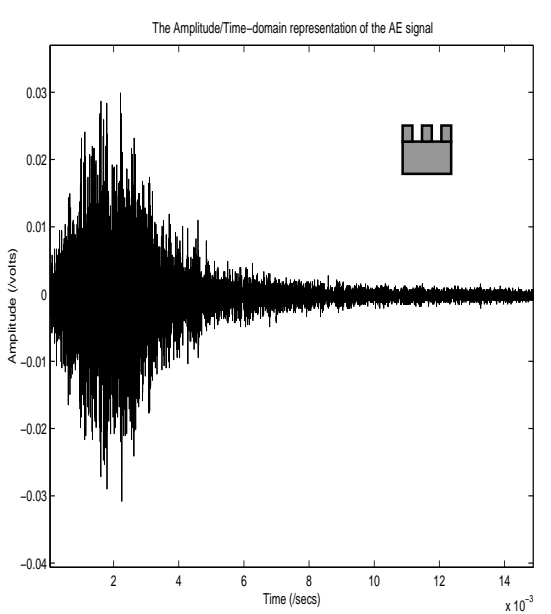

(a)

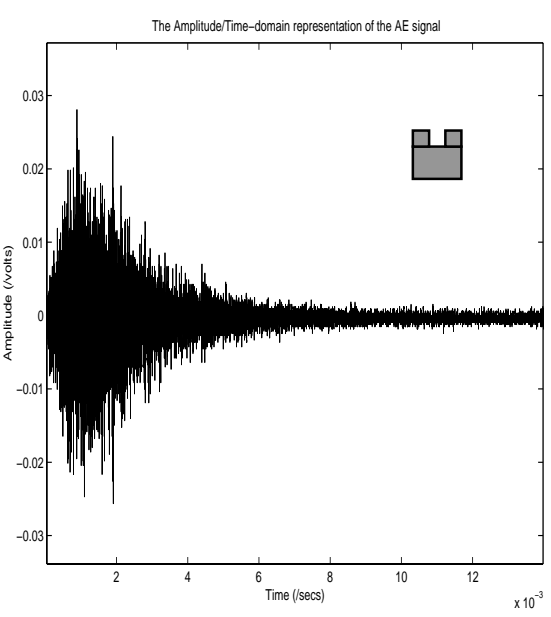

(b)

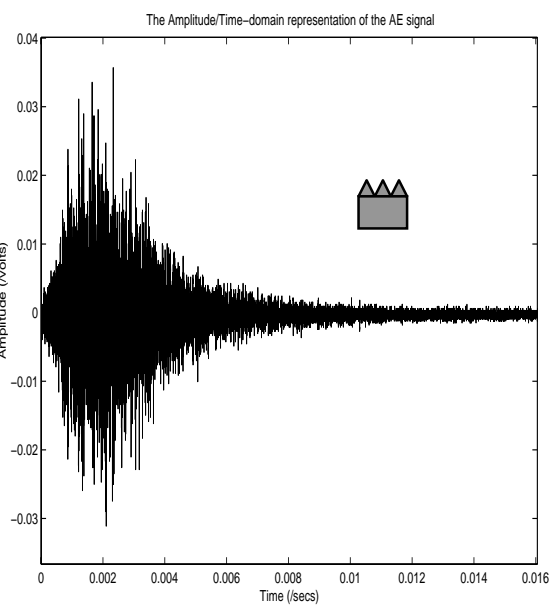

(c)

Figure 17: (a) Steel 2GFL signal (b) Steel 1GFL signal (c) Steel 2GKL signal

Again, it was found that there were no obvious differences in the amplitude levels of each signature class, as supported by figure-17. Again, this rendered classification based upon absolute values impractical. Moreover, it was found that the AR technique was not sensitive enough to distinguish between the different shape classes. This is demonstrated by taking five signals from each seal geometry class and effecting the same AR clustering technique. Figure-18 depicts the relatively poor seal shape 
Mechanical Systems and Signal Processing, 18 (4), pp. 849-868, 2004

clustering result achieved for an AR(20) model. Similarly, the clustering results observed using kurtosis were not adequate for resolving partial waveforms into separate clusters for different shaped fixtures.

Conversely, it was again found that the best classification results were achieved using the KS technique. Figure-19 depicts a dendrogram for the five AE signals considered from the three different seal shapes. This indicates that the KS statistic can separate the 2GKL seal from the other two types. However, it was consistently found that distinction between the 2GFL and the 1GFL seal signals was not achieved using the KS statistic. This proved to be the case no matter what combination of reference models (i.e. candidate distribution families as described in the Theory section) were used to evaluate KS values within the input feature vector.

This consistent lack of success in distinguishing between the 2GFL and 1GFL seals of the same material may be justified in terms of the physical rub mechanism. By closer inspection of the two seal types, it is observed that the contact surface areas of the 2GFL and 1GFL seals are exactly the same. Therefore during partial rubbing, an equivalent total flat area of each seal will come into contact with the rotating shaft. Hence, it is postulated that for such flat labyrinth shaped seals, a similar total number of asperity contacts occur during the partial rub duration, even though there might be some difference in localised pressure. Consequently, this results in a statistical similarity between the measured AE waveforms. 
Mechanical Systems and Signal Processing, 18 (4), pp. 849-868, 2004

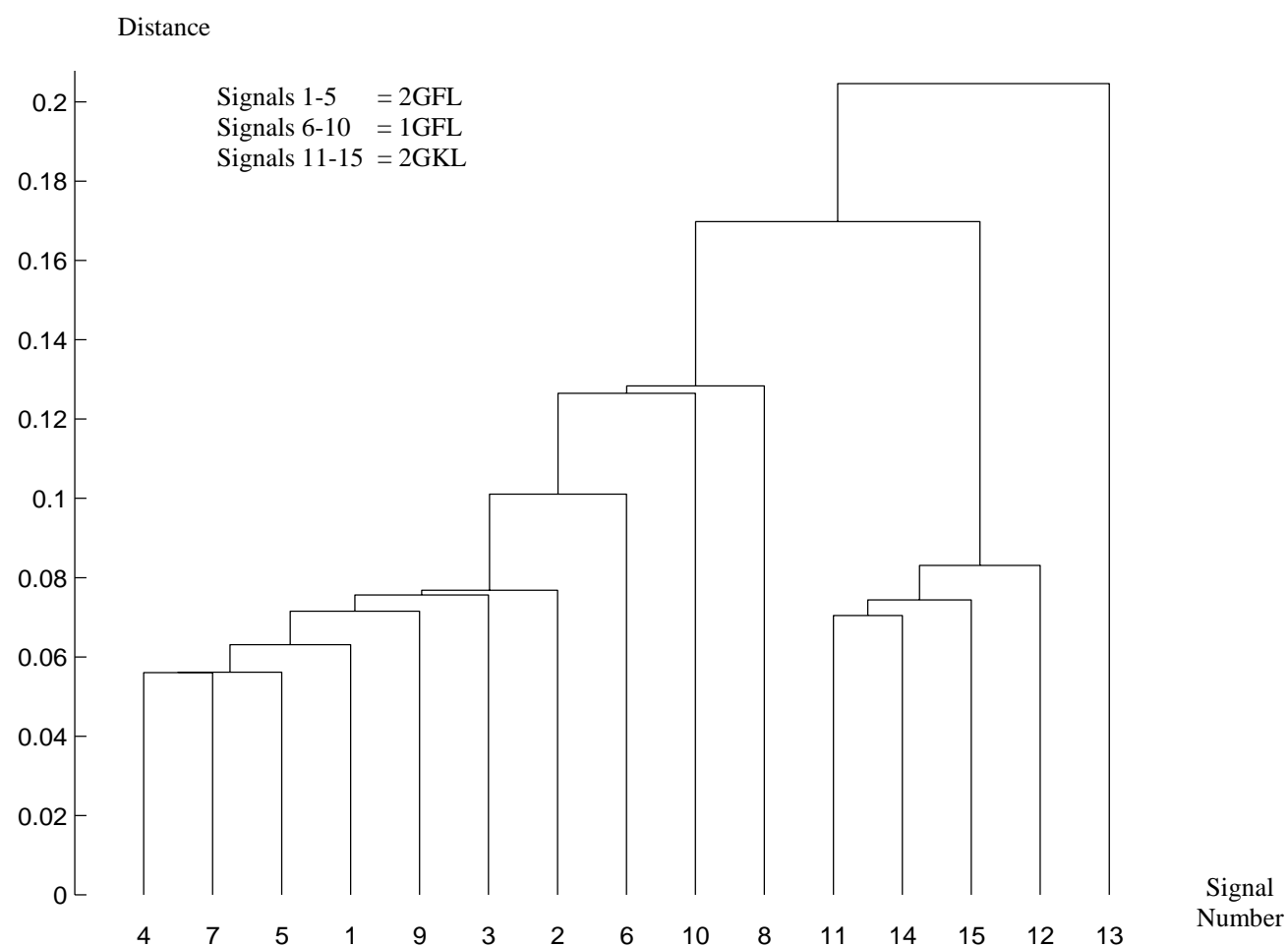

Figure-18: AR(20) Clustering for Steel 2GFL signals (1-5), Steel 1GFL signals(6-10), Steel 2GKL signals (11-15). 
Mechanical Systems and Signal Processing, 18 (4), pp. 849-868, 2004

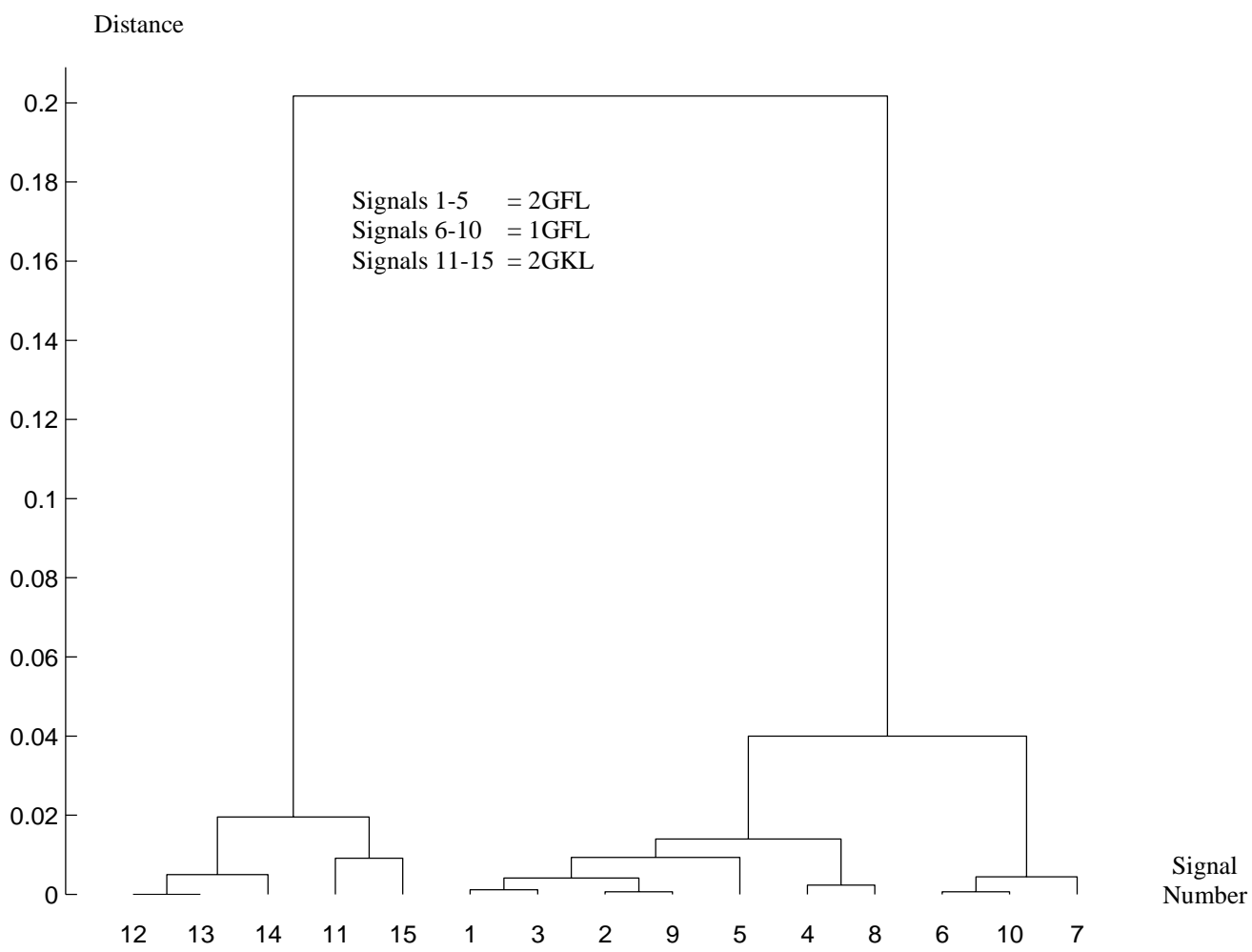

Figure 19: Gaussian KS-Clustering for Steel 2GFL signals (1-5), Steel 1GFL signals(6-10) Steel 2GKL signals (11-15).

\section{Conclusions}

This paper introduces the use of the Kolmogorov-Smirnov (KS) test statistic as a useful signal descriptor in AE analysis. The KS results presented via hierarchical dendrograms indicate the potential of this statistic in classification of partial rubbing on shafts of fast rotating machinery. Moreover, the success of KS classification has been shown when the measured AE rub signals were modulated by background noise from a real operational 500-MW turbine.

The KS technique offers a different and versatile way of probing the shape of the amplitude distribution function or CDF. It is noted that the KS statistic tends to be more sensitive around the medium value 
Mechanical Systems and Signal Processing, 18 (4), pp. 849-868, 2004

(i.e. where $\mathrm{P}(\mathrm{x})=0.5$ within the $\mathrm{CDF}$ ) and less sensitive at the extreme ends of the distribution, where $\mathrm{P}(\mathrm{x})$ approach either 0 or 1 . This is because the KS value does not have a probability distribution independent of amplitude (x). Therefore, whilst the KS statistic is good at finding shifts in the probability distribution, especially changes in medium value, it is not always so good at finding spreads, which more strongly affect the tails of the probability distribution [12]. Consequently, the KS statistic is robust and not too sensitive to variability in amplitude outliers as is the case with the fourth moment kurtosis. Thus it is concluded that the KS statistic provides a suitably stable feature for broadband AE signals that characterises the entire amplitude statistics.

Physical interpretation of individual AE signal waveforms from partial rubbing is difficult. However, it is suggested that the Gaussian derived KS statistic may be used to relate to the incident physical rub mechanisms. Specifically, it is considered that a form of the Central Limit Theorem can be applied. That is, a rub induced AE waveform, formed by the addition of a number of temporally close but independent asperity contacts, has a probability density function, which approaches the Gaussian distribution, as the number of contributors or AE sources increases. Consequently, it is postulated that absolute KS values for a Gaussian fit directly quantify the number of concentration of asperity events within the rub event, thereby inferring more detailed rub diagnosis such as the rub force or severity. Finally, it is noted that these classification results were achieved using a high-fidelity wideband AE transducer. However, it is realised that the KS technique could break down using low fidelity resonant devices. 
Mechanical Systems and Signal Processing, 18 (4), pp. 849-868, 2004

\section{References}

1. P.D. McFadden and J.D. Smith, (1983), “Acoustic Emission transducers for vibration monitoring of bearings at low speeds”, Report CUED/C-Mech. TR29, University of Cambridge, Cambridge.

2. I. Sato (1990), "Rotating machinery diagnosis with acoustic emission techniques”, Electrical Eng Japan, 110(2), 115-127

3. T. Holroyd and N. Randall (1992), "The Use of Acoustic Emission for Machine Condition Monitoring”, British Journal of Non-Destructive Testing, 35(2), 75.

4. D. Mba, and R. H. Bannister (1999), "Condition monitoring of low-speed rotating machinery using stress waves: Part 1 and Part 2”, Proc Inst. Mech. Engrs., 213, Part E, 153-185.

5. A Choudhury and N. Tandon (2000), “Application of Acoustic Emission techniques for the detection of defects in rolling element bearings”, Tribology International, 33(1), pp. 39-45.

6. CB Board (2000), Stress wave analysis of turbine engine faults, Aerospace Conference, IEEE Proceedings (Cat. No.00TH8484), vol.6., pp. p.79-93

7. W. Venkatesan (1996), "Detection and Modeling of Acoustic Emissions for Fault Diagnostics”, IEEE, 0-8186-7576-4/96.

8. C. Li (1989), “On-line bearing localized defect detection by pattern recognition analysis”, ASME Trans., J. Eng. Ind, 111, 331.

9. K. Ono and Q. Huang (1994), "Pattern recognition analysis of acoustic emission signals", Progress in Acoustic Emission, VII, Japanese Society for NDI, Tokyo, pp. 69-78

10. C.Chan (1985), “Classification of Acoustic Emission Signals Generated During Welding”, Journal of Acoustic Emission, 4(4) 115.

11. R. Melton (1982), “Classification of NDE waveforms with Autoregressive models”, Journal of Acoustic Emission, 1(4), 266. 
Mechanical Systems and Signal Processing, 18 (4), pp. 849-868, 2004

12. W. H. Press, S. A. Teulosky, B. P. Vetterling and W. T. Flannery (1993), Numerical Recipes in C, ISBN: 0521431085, pp620, Cambridge University Press

13. C. R. L. Murthy, B. Dattaguru, A.K. Rao (1987), “Application of pattern recognition concepts to acoustic emission signal analysis”, Journal of Acoustic Emission, 6(1), 19-28.

14. P.G. Bently (1988), “Acoustic emission measurement on PWR weld material with inserted defects using advanced instrumentation”, Journal of Acoustic Emission, 7(2), 59.

15. A. Anastassopoulos and Philippidis (1995), "Clustering Methodology for the Evaluation of Acoustic Emission from Composites”, Journal of Acoustic Emission, 13(1-2), 11.

16. R. Melton (1982), “Classification of NDE waveforms with Autoregressive models”, Journal of Acoustic Emission, 1(4), 266.

17. S.M. Kay, and S.L. Marple, Jr (1981), “Spectrum analysis - A modern perspective”, Proceedings of the IEEE, 69(11), 1380-1419.

18. J. Makhoul, (1975), “Linear prediction: A tutorial review”, Proc. of the IEEE, 63(4), 561-580. 
Mechanical Systems and Signal Processing, 18 (4), pp. 849-868, 2004

\section{Appendix}

\begin{tabular}{|c|c|c|c|c|c|c|c|c|c|c|c|c|c|c|c|}
\hline SIGNAL & $\begin{array}{c}\text { KS- } \\
\text { Gauss }\end{array}$ & $\begin{array}{l}\text { KS- } \\
\text { Rayl }\end{array}$ & $\begin{array}{l}\text { KS- } \\
\text { Exp }\end{array}$ & $\begin{array}{c}\text { KS- } \\
\text { Gauss } \\
\times 2 \\
\end{array}$ & RMS & Med & Kurt & $\begin{array}{l}\text { AR } \\
(1)\end{array}$ & $\begin{array}{l}\text { AR } \\
(2)\end{array}$ & $\begin{array}{l}\text { AR } \\
(3)\end{array}$ & $\begin{array}{l}\text { AR } \\
(4)\end{array}$ & $\begin{array}{l}\text { AR } \\
(5)\end{array}$ & $\begin{array}{l}\text { AR } \\
(6)\end{array}$ & $\begin{array}{l}\text { AR } \\
(7)\end{array}$ & $\begin{array}{l}\text { AR } \\
\text { (8) }\end{array}$ \\
\hline Steel-1 & 0.108 & 0.618 & 0.839 & 0.103 & 0.007 & 0.013 & 10.176 & -2.002 & 1.251 & 0.041 & -0.126 & -0.011 & -0.094 & 0.081 & 0.052 \\
\hline Steel-3 & 0.103 & 0.609 & 0.832 & 0.100 & 0.006 & 0.015 & 11.159 & -2.012 & 1.244 & 0.070 & -0.132 & -0.040 & -0.054 & 0.043 & 0.067 \\
\hline Steel-4 & 0.097 & 0.613 & 0.833 & 0.108 & 0.012 & 0.016 & 10.644 & -2.007 & 1.259 & 0.022 & -0.106 & -0.005 & -0.104 & 0.064 & 0.068 \\
\hline Steel-5 & 0.130 & 0.629 & 0.828 & 0.124 & 0.010 & 0.012 & 12.443 & -2.001 & 1.236 & 0.058 & -0.120 & -0.047 & -0.047 & 0.047 & 0.062 \\
\hline Steel-6 & 0.083 & 0.605 & 0.849 & 0.089 & 0.009 & 0.014 & 9.3301 & -1.973 & 1.180 & 0.099 & -0.130 & -0.039 & -0.055 & 0.048 & 0.065 \\
\hline Steel-8 & 0.098 & 0.615 & 0.834 & 0.097 & 0.008 & 0.016 & 10.462 & -2.010 & 1.263 & 0.032 & -0.117 & -0.027 & -0.068 & 0.052 & 0.065 \\
\hline Steel-9 & 0.103 & 0.614 & 0.836 & 0.104 & 0.006 & 0.014 & 11.377 & -2.020 & 1.271 & 0.041 & -0.119 & -0.048 & -0.042 & 0.039 & 0.066 \\
\hline Steel-10 & 0.107 & 0.617 & 0.819 & 0.107 & 0.010 & 0.017 & 11.894 & -2.003 & 1.233 & 0.068 & -0.121 & -0.035 & -0.068 & 0.051 & 0.067 \\
\hline Steel-11 & 0.104 & 0.618 & 0.821 & 0.102 & 0.010 & 0.014 & 10.951 & -2.005 & 1.245 & 0.051 & -0.130 & -0.011 & -0.074 & 0.040 & 0.073 \\
\hline Steel-12 & 0.088 & 0.598 & 0.806 & 0.086 & 0.005 & 0.024 & 9.953 & -1.975 & 1.198 & 0.066 & -0.114 & -0.016 & -0.107 & 0.092 & 0.052 \\
\hline Steel-13 & 0.110 & 0.624 & 0.796 & 0.111 & 0.006 & 0.018 & 10.109 & -1.987 & 1.208 & 0.079 & -0.119 & -0.048 & -0.053 & 0.052 & 0.062 \\
\hline Steel-14 & 0.107 & 0.616 & 0.794 & 0.107 & 0.006 & 0.019 & 9.958 & -1.998 & 1.221 & 0.080 & -0.134 & -0.039 & -0.057 & 0.056 & 0.058 \\
\hline Steel-15 & 0.119 & 0.621 & 0.805 & 0.113 & 0.012 & 0.018 & 11.467 & -1.995 & 1.237 & 0.049 & -0.121 & -0.016 & -0.091 & 0.076 & 0.056 \\
\hline Brass-17 & 0.052 & 0.583 & 0.877 & 0.059 & 0.006 & 0.013 & 8.056 & -2.015 & 1.209 & 0.097 & -0.098 & -0.070 & -0.059 & 0.045 & 0.063 \\
\hline Brass-18 & 0.063 & 0.582 & 0.872 & 0.066 & 0.006 & 0.015 & 8.793 & -1.984 & 1.143 & 0.126 & -0.084 & -0.029 & -0.143 & 0.089 & 0.058 \\
\hline Brass-19 & 0.043 & 0.576 & 0.893 & 0.042 & 0.006 & 0.014 & 7.090 & -2.028 & 1.236 & 0.082 & -0.095 & -0.087 & -0.023 & 0.009 & 0.078 \\
\hline Brass-20 & 0.054 & 0.570 & 0.882 & 0.059 & 0.006 & 0.014 & 7.494 & -1.979 & 1.118 & 0.163 & -0.090 & -0.068 & -0.089 & 0.049 & 0.073 \\
\hline Brass-21 & 0.042 & 0.576 & 0.896 & 0.042 & 0.001 & 0.014 & 6.662 & -2.010 & 1.192 & 0.121 & -0.109 & -0.069 & -0.060 & 0.042 & 0.067 \\
\hline Brass-22 & 0.053 & 0.578 & 0.873 & 0.048 & 0.007 & 0.016 & 7.880 & -1.991 & 1.155 & 0.142 & -0.110 & -0.062 & -0.070 & 0.045 & 0.067 \\
\hline Brass-23 & 0.063 & 0.583 & 0.867 & 0.062 & 0.007 & 0.014 & 8.362 & -1.998 & 1.170 & 0.133 & -0.106 & -0.079 & -0.041 & 0.022 & 0.075 \\
\hline Brass-24 & 0.059 & 0.577 & 0.873 & 0.055 & 0.007 & 0.015 & 8.032 & -2.017 & 1.218 & 0.079 & -0.080 & -0.075 & -0.058 & 0.036 & 0.069 \\
\hline Brass-25 & 0.053 & 0.581 & 0.874 & 0.050 & 0.006 & 0.015 & 7.236 & -2.041 & 1.264 & 0.073 & -0.118 & -0.056 & -0.058 & 0.045 & 0.060 \\
\hline Brass-26 & 0.061 & 0.580 & 0.882 & 0.056 & 0.007 & 0.014 & 8.643 & -2.015 & 1.206 & 0.103 & -0.101 & -0.066 & -0.065 & 0.046 & 0.063 \\
\hline Brass-27 & 0.064 & 0.587 & 0.882 & 0.065 & 0.009 & 0.013 & 8.608 & -1.996 & 1.162 & 0.141 & -0.103 & -0.088 & -0.036 & 0.022 & 0.075 \\
\hline Brass-28 & 0.042 & 0.578 & 0.886 & 0.044 & 0.005 & 0.014 & 7.448 & -2.017 & 1.217 & 0.095 & -0.109 & -0.051 & -0.074 & 0.049 & 0.064 \\
\hline Brass-29 & 0.058 & 0.584 & 0.872 & 0.064 & 0.005 & 0.014 & 8.196 & -2.006 & 1.188 & 0.110 & -0.091 & -0.080 & -0.046 & 0.024 & 0.075 \\
\hline Brass-30 & 0.044 & 0.580 & 0.888 & 0.051 & 0.006 & 0.014 & 7.700 & -1.986 & 1.143 & 0.143 & -0.099 & -0.043 & -0.120 & 0.078 & 0.061 \\
\hline
\end{tabular}

